\title{
Gait Characteristics, Range of Motion, and Spasticity Changes in Response to Massage in a Person with Incomplete Spinal Cord Injury: Case Report
}

\author{
Christine Manella, PT, MT, ${ }^{1}$ Deborah Backus, PhD, $\mathrm{PT}^{2}$ \\ ${ }^{1}$ Multiple Sclerosis/Spinal Cord Injury Programs, Shepherd Center, and ${ }^{2}$ Spinal Cord Injury Research, Crawford Research \\ Institute, Shepherd Center, Atlanta, GA, USA
}

Purpose: Our study set out to measure the effect of a specific routine of massage on gait characteristics, range of motion, and spasticity in a person with incomplete spinal cord injury.

Methods: This descriptive, pre-post case study, conducted at the outpatient program of a rehabilitation facility, used neuromuscular techniques in massage for a 42-year-old man with incomplete chronic C5 spinal cord injury. The massage was applied to the iliopsoas, triceps surae, and hamstring muscle groups for 3 consecutive days.

Main Outcome Measures: Pre-and post-intervention testing included standard goniometric measurement of joint range of motion in the lower extremities, spasticity evaluation using the modified Ashworth scale, and evaluation of gait characteristics using GAITRite Walkway (CIR Systems, Havertown, PA, USA) pressure mapping for ambulation time, cadence, velocity, stride length, base of support, and single- and double-limb support.

Results: After the therapeutic intervention, the following gait changes were demonstrated: increase in velocity and cadence of gait, decrease in ambulation time, increase in stride length, and improvements in the percentages of the swing and stance phases of the gait cycle.

Conclusions: Specific application of massage therapy influenced gait speed, stride length, and swing and stance phase percentages in one person with incomplete spinal cord injury. Further study is warranted to determine the extent to which massage may affect musculoskeletal and neural impairments that limit gait in people with incomplete spinal cord injury, and the method or routine whose application will yield the most benefit.

KEYWORDS: Massage, spinal cord injury, GAITRite, gait characteristics

\section{INTRODUCTION}

Massage techniques are not frequently used in traditional neurological rehabilitation settings. The present case report, concerning a person with incomplete spinal cord injury, reflects on the possible integration of massage to complement rehabilitation goals.

Approximately 259,000 people with a spinal cord injury (SCI) live in the United States ${ }^{(1)}$. Of those recently injured, approximately $30.1 \%$ are classified as having incomplete tetraplegia ${ }^{(1)}$. Some of those with incomplete tetraplegia are classified " $D$ " on the American Spinal Injury Association impairment scale (AIS). Because of preservation of some motor and sensory function below the level of injury, these people often regain the ability to ambulate.

An AIS(D) cervical injury leads to motor and sensory impairments in the upper limbs, lower limbs, and trunk, with the greatest effects occurring in the upper half of the body. The ability of muscle to function against gravity is preserved in more than half of all muscle groups below the neurological level of injury. However gait patterns in this population often reflect incoordination of muscle action, leading to inefficient gait, with high-level energy consumption $^{(2)}$. Although able to ambulate, these people are often unable to isolate specific muscle patterns to ambulate at functional speeds and without significant gait deviations. Gait impairments in people with incomplete SCI are not only induced by alterations in motor control, but also by muscle weakness, decreased range of motion (ROM), and decreased muscle extensibility ${ }^{(3,4)}$.

\section{Biomechanics of Gait}

Gait is a complicated activity, with demands for complex coordination of eccentric and concentric muscle activation, and requirements for varying degrees of ROM timed within the ambulation cycle. Gait deviations commonly observed after SCI include inadequate hip extension in stance, limited hip and knee flexion in swing, excessive plantar flexion, and impaired foot contact at initial stance ${ }^{(5)}$. Other characteristics of gait after incomplete SCI are a narrow base of support and decreased gait speed compared with those in able-bodied people ${ }^{(6)}$, shortened step length, and increased duration of double-limb support $^{(7,8)}$. 
Three muscle groups are often cited as contributors to impaired gait patterning in populations with neurological dysfunctions:

- Triceps surae

- Hamstrings

- Iliopsoas

Dysfunction in these three muscle groups can lead to the aforementioned compensatory mechanisms that result in inefficient gait in people with incomplete SCI. Specifically, poor timing, overactivity, or underactivity in these muscles can lead to predictable alterations in the kinematics of gait. Within the phases of the gait cycle, muscle dysfunction in these groups can cause specific gait deviations. The phases of gait include the loading response, midstance, terminal stance, pre-swing, initial swing, mid-swing, terminal swing, and push-off.

\section{Muscle Activity Changes Resulting in Gait Dysfunction}

During stance limb loading, initial contact can present with foot flat or forefoot contact because of constant activation of the triceps surae. Overactivity in the triceps surae group negates the ability for the required pre-tibial activity to occur, interferes with foot clearance, creates poor shock absorption, and contributes to limited knee flexion during weight acceptance $^{(9)}$.

During midstance, foot flat contact impairs tibial motion, leading to a shortened step length. Additionally there may be compensation with premature heel-off, knee hyperextension, or forward trunk lean ${ }^{(10)}$. Normally during midstance, the hip extends to a neutral position, but by terminal stance, the hip should reach 20 degrees of hyperextension. Tightness or spasticity of the iliopsoas muscle may limit hip extension in the midstance and terminal stance phases of gait. By limiting hip extension in the hypertonic state, the iliopsoas can also inhibit gluteus maximus function ${ }^{(11)}$ and contribute to an anterior pelvic tilt, causing increased energy cost to the hip and trunk extensors and to the overall energy expenditure for gait $^{(9)}$.

Heel rise, forefoot rocker action, and additional ROM to 10 degrees of ankle dorsiflexion (DF) generate high torque demands in terminal stance. This combination of events in terminal stance allows initiation of the contralateral step. Adequate ankle DF ROM is therefore essential to achieve proper gait mechanics in this phase.

During the swing phase of gait, calf muscle activity should ease, allowing the pre-tibial musculature to activate and move the ankle to neutral position ${ }^{(9,10)}$. Hypertonicity of the gastrocnemius and soleus muscles may inhibit tibialis anterior action during terminal stance when demands for DF torque are greatest. Hamstring spasticity and shortened states can also lead to inadequate knee extension in terminal stance, decreased step length, and interference with initial contact of the foot.

In normal walking, the activity of the biceps femoris short head peaks during pre-swing, resulting in knee flexion up to 60 degrees. The entire hamstring group then becomes active in mid-swing and terminal swing to decelerate the thigh as the quadriceps contracts actively to achieve full knee extension at initial contact ${ }^{(9)}$. Shortening of the hamstring group by spasticity, as is often seen in people with SCI, can inhibit quadriceps function and the eccentric lengthening needed for control of forward motion of the thigh. Additionally, iliacus muscle activity should peak in pre-swing, aiding in limb advancement ${ }^{(9)}$. A shortened, inhibited iliacus will therefore impair that function.

These impairments that result in gait deviations after SCI often are not alleviated by simple strengthening of the muscles. Clinical practice typically includes reducing altered tone and improving and increasing ROM, under the assumption that these interventions may lead to beneficial effects on gait by assisting in controlling the relevant muscles. Another potential intervention is massage, which may lengthen the muscle tissue, leading to increased extensibility and flexibility and improved muscle control.

\section{Massage in Rehabilitation}

One of the most widely accepted uses and focuses of massage is the reduction of functional limitations resulting from musculoskeletal impairments ${ }^{(12-14)}$. Massage is also used as a tool in muscle recovery from fatigue for specific muscle groups ${ }^{(15,16)}$. Although some modest literature supports the use of massage to reduce musculoskeletal impairments in the able-bodied population $^{(17-19)}$, little available evidence supports this approach in people with SCI. The evidence that does exist suggests that people with SCI seek massage for the management of pain ${ }^{(20-22)}$. However, as will be discussed shortly, there is emerging evidence that massage may effect changes in musculoskeletal and neural functioning not only in the able-bodied but also in the neurologically impaired population.

Diego and colleagues ${ }^{(23)}$ demonstrated improvements in ROM and strength of the upper limbs after application of a set routine of massage and stretching in people with chronic (more than 1 year) cervical SCIs, compared with an exercise group. That study concentrated on upper limb function and did not address gait. In addition, it did not specify whether this group of people with SCI had complete or incomplete injuries, which is an important consideration when evaluating the effectiveness of massage. For instance, compared with people having complete injury, people with incomplete SCI are known to have greater issues with disordered movement control, and 
the latter group therefore experience impairments such as abnormally increased or decreased muscle activity, which may respond differently to massage interventions.

Massage has been shown to affect the neural influence on muscles. In neurologically healthy participants, Morelli and colleagues ${ }^{(24,25)}$ found reductions in H-reflex peak-to-peak amplitudes during a period of 3 minutes of petrissage to the triceps surae group (compared with control conditions), reflecting a reduction in motor neuron excitability that was statistically significant across all participants. In another study in a group of healthy adults, Morelli et al. ${ }^{(26)}$ further ruled out the effects of skin temperature, nerve conduction velocity, and antagonist recruitment as causes of decreased H-reflex amplitude recordings, concluding that the decrease was in response to 6 minutes of petrissage to calf muscles. That line of study was expanded by Goldberg et al. ${ }^{27)}$, who demonstrated the effectiveness of 3 minutes of one-handed petrissage on the reduction of H-reflex in the triceps surae muscle of healthy adults and of people with traumatic SCI (complete and incomplete injuries) above the lumbar segments. Those authors found that more than half the members of each population demonstrated a decrease in H-reflex amplitude (average: $27 \%$ ). Applying massage techniques in the SCI population, members of which are known to have deficits related to modulation of reflexes, may lead to positive changes.

Other authors have demonstrated the effect of massage on neural, musculoskeletal, or functional performance change in populations with various neurological impairments. In people with multiple sclerosis who presented with spasticity, Brouwer and Sousa de Andreade ${ }^{(28)}$ showed decreases in alpha motor neuron excitability in the triceps surae motor pool in response to 3 minutes of stroking over the posterior rami region - a reduction that lasted up to $30 \mathrm{~min}-$ utes post intervention. Macgregor and colleagues ${ }^{(29)}$ reported decreased stretch reflexes, improvements in gross motor function, and improvements in the ability to perform alternating movements in the ambulatory adolescent cerebral palsy population. Those improvements were obtained in response to application of a standardized sequence of Swedish massage strokes applied twice weekly over 5 weeks. Hernandez-Reif et al. ${ }^{(30)}$ also found improved hip extension ROM, improved gross motor function scores, and reduced muscle tone in the upper extremities for a group of 22 children of preschool age with cerebral palsy who received a set of massage sequences twice a week for 12 weeks in addition to traditional care.

Promotion, through massage, of proper lengthtension relationships in spastic muscles may encourage proper firing patterns ${ }^{(31,32)}$ and decrease motor-neuron excitability ${ }^{(33,34)}$, which may improve gait performance. Those findings support the hypothesis that massage can directly influence the neural components of muscle control in people with neurological injury or disease, including those with SCI.

Recently, in an acute rehabilitation setting, subjective reports of improved ease of movement post massage were related by the patients and the physical therapists (PTs) involved in a body-weight-supported treadmill training program for people with incomplete SCI. That evidence, taken in concert with the foregoing findings, leads to the hypothesis that massage can improve musculoskeletal and neural impairments affecting gait and may improve gait characteristics in general in people with incomplete SCI. Specifically, massage was expected to improve ROM and to decrease spasticity of the treated muscles, and therefore lead to improvements in gait parameters (that is, cadence, velocity, and ambulation time). Temporary or lasting changes in gait elicited by massage may provide rehabilitation staff with the opportunity to optimize improvements in ROM and muscle activation as an integrated part of standard care. In addition, changes in gait characteristics for this population may result in improved participation in life through improved balance, gait speed, and decreased dependence on assistive devices.

For the present case study, we set out to evaluate the effects of a massage intervention on ROM, spasticity, and gait characteristics in one person with an incomplete cervical spinal cord injury. We expected that massage would lead to temporary improvement in muscle length and control and result in improvements in gait.

\section{METHODS}

\section{Profile of Client}

For the present study, we recruited a 42-year-old man from a convenience sample of patients at a model SCI rehabilitation hospital. This specific participant, who had experienced a C5 incomplete SCI 23 months earlier, was chosen because the authors felt that he presented with gait deviations and posture common to many people with central-cord SCI. Ethics approval was received from the internal research review board, and the patient gave informed consent before initiation of study activities.

This participant's SCI had been sustained after a syncope episode of unknown cause; he had been in the bathroom and consequently hit his head on a bathroom fixture. He did not originally undergo cervical fixation, but was treated conservatively with a Philadelphia collar by an orthopedic surgeon. He underwent anterior cervical stabilization surgery approximately 6 months after the initial injury.

The injury was classified C5 AIS(D) using the International Standards for Neurological Classification of Spinal Cord Injury and the AIS ${ }^{(35)}$. During the 23 months since the initial injury, the participant 
had completed inpatient rehabilitation, bodyweightsupported treadmill training, and outpatient occupational therapy. He had previously received massage therapy during his inpatient rehabilitation, but he had not received any massage for the 6 months preceding the start of the present study. He was attending traditional physical therapy sessions twice weekly during the intervention period, and those sessions did not introduce any new modalities during the intervention week. He did not change any medications over the course of the study. He was ambulating full-time, using a single forearm crutch in the right upper limb. His goals were to normalize his gait pattern, to walk without an assistive device, and to experience increased ease of movement of his legs during walking.

\section{Evaluation}

Two licensed PTs performed outcome assessments pre-intervention for baseline data collection, post-treatment on each of the intervention days, and immediately after completion of the intervention period. The PTs were blinded to the order in which the interventions were applied.

The assessments included

- ROM. The PTs collected measurements using standard goniometry for assessment of hip extension, straight leg raise (SLR), and DF.

- Modified Ashworth scale (MAS) ${ }^{(36)}$. The MAS rates severity of tone on a scale from 0 to 4 (Table 1). It was used to assess overall muscle tone in the study participant. The test is performed in the supine position; the practitioner passively moves the limb at the speed of gravity. The speed of gravity is defined as the same speed a non-spastic limb would drop from a held position. Although the validity and reliability of this scale in the SCI population is debatable ${ }^{(37)}$, the MAS is typically the

TABLE 1. Grading Scheme for Modified Ashworth Scale(36)

Score Type of motion resistance observed (per movement)

0 No increase in muscle tone throughout flexion or extension movement

1 Slight increase in muscle tone manifested by a catch and release at the end range of motion when the limb is moved into flexion or extension

1+ Slight increase in muscle tone, manifested by a catch, followed by minimal resistance throughout the remainder $(<50 \%)$ of range of motion

2 More marked increase in muscle tone through most of the range of motion, but affected part is easily moved

3 Considerable increase in muscle tone; passive movement difficult

4 Affected part is rigid in flexion and extension tool of choice in therapy settings, given the labor and cost intensity of directly measuring spasticity with more objective tools, such as electromyography. The MAS was chosen for the present study because of ease of application and ability to apply the MAS directly after delivery of an intervention. The PTs collected data on lower extremity spasticity for bilateral hip flexion, abduction, internal and external rotation, knee flexion and extension, and DF. All measures were taken with the participant in supine position on the massage table.

- GAITRite Walkway (CIR Systems, Havertown, PA, USA) pressure mapping. The GAITRite Walkway system extrapolates data on step length, stride length, cadence, gait velocity, and single- and double-limb support. The GAITRite system creates these data by calculating spatial and temporal parameters in response to sensor activation as a person walks across the walkway (Figure 1). For each trial in the present study, the participant walked across the walkway, beginning at $152 \mathrm{~cm}$ (60 inches) from the start, using his normal assistive device of single forearm crutch to generate pressure map data on gait. The data were also analyzed using the GAITRite Walkway Analysis System (measurements defined by CIR Systems).

One PT collected data in the same order (range of motion, MAS, GAITRite) within the same 1-hour timeframe each day. Measurements of ROM and spasticity were all taken on the massage table on which treatment was provided during the intervention phase.

One PT performed observational gait assessment during the first session to provide clinical insight into

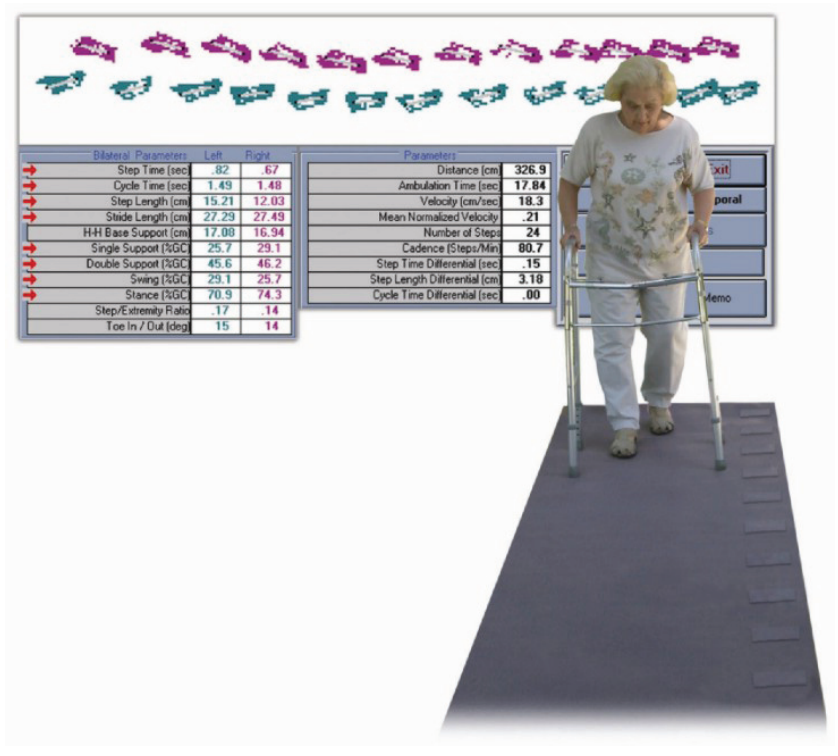

FIGURE 1. GAITRite (CIR Systems, Havertown, PA, USA) electronic walkway and pressure map. 
the participant's gait deviations and impairments. The gait dysfunction were characterized as follows:

- Excessive forward lean of the trunk throughout the gait cycle

- Shorter step and stride length for height

- Decreased gait velocity

- Narrowed base of support

- Decreased hip extension in late stance

- Decreased hip and knee flexion in swing phase

- Decreased heel strike at initial contact

- Posturing in left upper limb into flexion pattern throughout gait

Postural evaluation revealed tight iliopsoas musculature bilaterally (left greater than right), as demonstrated by forward trunk lean; anterior rotation of the left (compared with the right) ilium; rotation of the thoracic cage to right in the transverse plane, with left shoulder forward; intermittent recurvatum of both knees, depending on weight shift. The left upper limb was held in shoulder elevation, with elbow flexion and internal rotation.

\section{Intervention}

After baseline measures were collected, the participant underwent massage treatments each day for 3 sequential days. The three treated muscle groups were chosen by the primary investigator (triceps surae group, hamstrings, and iliopsoas) given their biomechanical effect on gait and the participant's gait evaluation. The goals of treatment for each muscle group were

- improvement in activation of the gluteus maximus, quadriceps, and anterior tibialis muscles by treating their shortened, antagonist counterparts. Justification: Treatment of a shortened, contracted muscle leads to inhibition of the antagonist ${ }^{(38)}$; thus, lengthening the contracted muscle should lead to improved activation of the agonist.

- improvement in muscle flexibility by increasing ROM of the affected joints, leading to ease of movement. Justification: Improved ROM may lead to increases in step length (hamstrings), improvements in heel contact (triceps surae), and increases in gait speed with ease of motion in swing (iliopsoas).

- reduction in trigger points to improve the lengthtension relationship and consequently the ability to generate force and velocity within the muscles. Justification: Trigger points may propagate spasticity, leading to sensitization of the reflex arch, and may contribute to impaired length-tension relationships ${ }^{(39)}$.

- improvement in the firing pattern in the affected muscle groups by reduction of spasticity. Justification: A decrease motor neuron excitability may lead to reduction in spasticity and improved firing pattern. People with incomplete SCI who also exhibit spasticity would therefore benefit from specific massage treatment to muscle groups displaying hypertonicity ${ }^{(27,31,32)}$.

All treatments were provided by a licensed massage therapist (MT). The MT was a 41-year-old woman with 11 years of experience in massage therapy. The massage interventions were based on routines outlined in Chaitow and DeLany's 2002 Clinical Application of Neuromuscular Techniques ${ }^{(11)}$. Tables 2, 3, and 4 outline the details of each routine. The routines are

TABLE 2. Massage Routine for Triceps Surae Group Adapted from Chaitow and DeLany $2002^{(11)}$

Position Participant prone on massage table, with foot resting on a 4-inch foam wedge

Practitioner at level of foot

Method 1. Gliding strokes applied cephalically to the most lateral segments of the gastrocnemius with palmer surface of hand. Therapist moves hand medially $1-2$ inches, and gliding strokes are repeated. Strokes applied segmentally, and in lateral-to-medial fashion in 5 sections until entire posterior surface of leg muscle treated. A total of 40 strokes are applied.

2. Areas identified by therapist as restricted, or that reproduce pain pattern, are re-treated with thumb compression of $10 \mathrm{~s}$. The therapist identifies 5 areas for treatment.

3. Knee flexed to approximately 45 degrees of flexion. Belly of gastrocnemius lifted by pincer grip and manipulative compression applied to the medial and lateral portion from proximal attachments towards Achilles tendon for a total of 2 minutes.

4. Ankle positioned in slight dorsiflexion for the doublethumb gliding technique. Thumbs positioned deep to the gastrocnemius, and opposite each other on the sides of the leg. Practitioner glides thumbs proximally while simultaneously pressing them towards each other. Repeat glides 8 times.

5. Knee passively flexed to $70-90$ degrees. Palpation of the lateral head of the gastrocnemius made by placing thumb between biceps femoris tendon and iliotibial band, directly into the lateral condyle of the femur. Static pressure for $10 \mathrm{~s}$ applied at insertion. Procedure repeated for medial head by working just medial to the semimembranosus and semitendinosus tendon into the medial epicondyle of femur. Static pressure for $10 \mathrm{~s}$.

6. Leg repositioned on the wedge. With thumb pressure toward each other, either side of the tendon, the double-thumb gliding technique (as in step 5) is applied while entrapping the tendon. Glides are applied proximally 10 times, calcaneus to mid-calf.

7. Glides applied cephalically to posterior surface of tendon proximally 10 times with thumbs-from calcaneus insertion to mid-calf.

8. Achilles tendon displaced medially, then laterally to gain exposure to the anterior surface of the tendon. Static pressure is applied to each side for $10 \mathrm{~s}$ with fingertip pressure. 
Table 3. Massage Routine for Iliopsoas Group Adapted from Chaitow and DeLany $2002^{(11)}$

$\begin{array}{ll}\text { Position } & \begin{array}{l}\text { Participant supine, knees flexed, with ipsilateral knee } \\ \text { resting on practitioner's knee } \\ \text { Fingers of both practitioner's hands placed against me- } \\ \text { dial aspect of anterior superior iliac spine and directly } \\ \text { against interior surface of ilium }\end{array} \\ \text { Method } & \text { 1. Fingers slide slowly and firmly along interior wall } \\ \text { of the ilium while contacting and pressing iliacus in } \\ \text { to bony structure with static pressure of 10-s incre- } \\ \text { ments, followed by friction, if tolerated. Fingers } \\ \text { move from the iliac crest to inguinal ligament while } \\ \text { moving posteriorly in 1-inch increments. Therapist } \\ \text { maintains direct contact entire treatment time. Total } \\ \text { time: } 10 \text { minutes. }\end{array}$

Reposition Participant's leg is placed on 6-inch foam roll placed under the knee (foam roll was used for placement because participant lacked the ability to hold knees in flexion throughout the routine)

Method 2. Fingertip of practitioner's hands positioned vertically at lateral edge of rectus abdominis, approximately 2 inches lateral to umbilicus. A steady, painless pressure is applied with slight rotary movements until contact with psoas is reached. Position is checked by having patient initiate active-resistive hip flexion against practitioner's elbow. Once location is confirmed, therapist applies light, direct compressive pressure into psoas in 1- to 2 -inch increments for the length and width of the muscle. When tender points are noted, sustained pressure is held for $10 \mathrm{~s}$. Tender-point treatment limited to 5 locations. Total time: 15 minutes.

3. Iliopsoas tendon is located just inferior to inguinal ligament and lateral to femoral pulse (both structures located). Static pressure for 3 bouts of $10 \mathrm{~s}$, followed by friction for $10 \mathrm{~s}$ to the tendon.

well documented and reproducible, and were chosen with the intent of using multiple physiological pathways for improved muscle function.

Modifications to the routines were made to exclude passive and myofascial stretching because changing the participant's position on the table may have, unintentionally, evoked a spasm or caused confounding effects of stretching on the results. The variety of strokes within the routines not only mimic "real world" application of massage, but also use a variety of mechanisms to achieve changes in the objective measures. Gliding and kneading strokes mimic slow passive stretch, which may help to reset muscle tonus ${ }^{(40)}$. Static and ischemic pressure help to reduce trigger points and to reset shortened sarcomere length ${ }^{(31)}$. In some steps, the number of strokes was increased from the original format. In addition, the therapist was required to quantify the total time of stroke application, which the original outline of treatment does not require ${ }^{(11)}$.

Routines were applied to the bilateral lower extremities and were timed to match in total time for
TABle 4. Massage Routine for Hamstring Group (Biceps Femoris, Semimembranosus, Semitendinosus) Adapted from Chaitow and DeLany $2002^{(11)}$

Position Participant prone on massage table, feet supported on a 4-inch foam wedge

Practitioner on lateral side of hamstring being treated

Method 1. Lateral aspect of muscle group identified through resisted knee flexion.

2. Gliding strokes with lubrication applied distally to proximally beginning at most lateral and posterior aspect of thigh and moving medially to encompass the entirety of the biceps femoris group. Therapist uses palmer surface of hand and applies 8 strokes cephalically and segmentally in 3 sections, laterally to medially (a total of 24 strokes applied until entirety of muscle group is treated).

3. Areas identified by therapist as restricted, or that reproduce pain pattern, are retreated with thumb compression of $10 \mathrm{~s}$. The therapist identifies 5 areas for treatment.

4. Proximal attachment of hamstrings identified by hip extension and knee flexion while palpating the ischial tuberosity.

5. Compression with thumbs applied for 3 bouts of $10 \mathrm{~s}$, followed by medial-lateral friction for $10 \mathrm{~s}$ on the insertion of ischial tuberosity.

6. Knee is moved into passive flexion to identify distal attachment, pincer compression used to grasp tendons individually. Manipulative compression is applied from most proximal palpable lateral tendon to tibia/fibula for 2 minutes. Cross-friction is applied at insertion for $10 \mathrm{~s}$.

Reposition Practitioner to contralateral side of the table

Method 7. Gliding strokes with lubrication applied distally to proximally, beginning at most medial, posterior aspect of thigh and moving laterally, encompassing the entirety of the semimembranosus and semitendinosus. Therapist uses palmer surface of hand and applies 8 strokes cephalically and segmentally, moving medially to laterally in 3 sections until entirety of muscle group is treated (total of 24 strokes applied until entirety of muscle group is treated).

8. Areas identified by therapist as restricted, or that reproduce pain pattern, are re-treated with thumb compression of $10 \mathrm{~s}$. The therapist identifies 5 areas for treatment.

9. Manipulative compression is applied in same manner as in step 7, to semimembranosus and semitendinosus insertion on tibia. For 2 minutes, cross-friction is applied at insertion for $10 \mathrm{~s}$.

each side on each treatment day. To track individual outcome in each muscle group and to determine whether treatment of any single group produced a greater therapeutic effect, just one muscle group was treated each day.

For all treatments, the MT timed each routine so as not to exceed 30 minutes on each lower extremity, 
each ischemic hold time being self-timed with a standard watch. Within 15 minutes of each treatment session being completed (days 2, 3, 4), a licensed PT collected data for ROM, MAS, and gait characteristics. See Table 5 for a timeline of the data collection and treatments provided.

Data for ROM and MAS were manually recorded; GAITRite data were saved electronically. The GAITRite Data were collected by a trained technician.

\section{RESULTS}

Table 6 reports the changes in ROM over the course of the study. No significant changes in ROM were observed for SLR throughout the study. Right hip extension showed a positive gain in range on days 1 and 3, and left hip extension showed a positive gain on day 3 . On post-intervention measures, hip extension demonstrated positive gains bilaterally. Measurements of DF were relatively unchanged on the right except after the day 3 intervention, when a negative measure was noted. Left DF demonstrated

TABLE 5. Timeline of Data Collection and Intervention

\begin{tabular}{ll} 
Time & Activity \\
\hline Pre-test & $\begin{array}{l}\text { Data collected } \\
\text { No massage intervention }\end{array}$ \\
Day 1 & $\begin{array}{l}\text { Treatment of the triceps surae with the massage routine } \\
\text { outlined in Table } 2\end{array}$ \\
& $\begin{array}{l}\text { Participant was repositioned once during the routine } \\
\text { because of a spasm of the right lower extremity into knee } \\
\text { flexion during treatment of the ischemic area. }\end{array}$ \\
Day 2 & $\begin{array}{l}\text { Treatment to the iliopsoas group as outlined in Table } 3 \\
\text { Day 3 }\end{array}$ \\
Post-test & $\begin{array}{l}\text { Data collected } \\
\text { No massage intervention }\end{array}$
\end{tabular}

a negative measure on day 2 and a less than 5-degree change on days 1 and 3 . Left DF showed a positive effect on the post-intervention assessment.

No consistent change in MAS score was observed in either lower extremity at any joint.

Changes in gait parameters were observed over the course of the study (Table 7). Stride length in the GAITRite system is measured along the line of progression between the heel points of two consecutive footprints of the same foot, left to left and right to right. Increases on both the left $(15 \%)$ and the right side $(13 \%)$ were noted. The greatest change in length occurred on day 3 , with an $8.09 \mathrm{~cm}$ gain on the left and an $8.18 \mathrm{~cm}$ gain on the right.

Velocity of ambulation (distance traveled divided by ambulation time) increased by $24 \%$ from pre-test to post-test. Cadence (steps per minute) increased over the course of the study by $9 \%$, with the highest gains occurring on day 2 .

Ambulation time (time elapsed between first contact for the first and last footfalls across the pressure map) declined by $17 \%$ over the course of the study, with the greatest decrease noted on day 3 .

No consistent changes were observed in the base of support or in the single- or double-limb support percentages. Changes in the swing and stance phases of gait are highlighted in Table 8 and Figure 2. The percentage of swing increased and the percentage of stance time decreased throughout the gait cycle.

\section{Participant Reports}

The participant reported greater fluidity of gait and improved heel strike 3 days post triceps surae treatment. The participant said that gait was less strenuous for 5 days after the last intervention day. No pain or muscle soreness was reported throughout the study. Although the participant's goal of ambulating without an assistive device was not achieved, his goals of a more normalized gait pattern and increased ease of movement were achieved.

TABLE 6. Range-of-Motion Measurements

\begin{tabular}{|c|c|c|c|c|c|c|}
\hline \multirow[t]{2}{*}{ Movement } & \multicolumn{5}{|c|}{ Motion (degrees) } & \multirow{2}{*}{$\begin{array}{l}\text { Magnitude } \\
\text { of change }\end{array}$} \\
\hline & Pre-test & Day 1 & Day 2 & Day 3 & Post-test & \\
\hline \multicolumn{7}{|c|}{ Straight leg raise } \\
\hline Right & 58 & 56 & 60 & 54 & 61 & 3 \\
\hline Left & 50 & 53 & 52 & 53 & 54 & 4 \\
\hline \multicolumn{7}{|c|}{ Hip extension } \\
\hline Right & $(-) 11$ & $(-) 4$ & $(-) 10$ & 6 & 6 & 17 \\
\hline Left & $(-) 8$ & $(-) 7$ & $(-) 8$ & 14 & 9 & 17 \\
\hline \multicolumn{7}{|c|}{ Ankle dorsiflexion } \\
\hline Right & $(-) 12$ & $(-) 12$ & $(-) 12$ & $(-) 18$ & $(-) 11$ & 1 \\
\hline Left & $(-) 10$ & $(-) 7$ & $(-) 7$ & $(-) 11$ & $(-) 2$ & 8 \\
\hline
\end{tabular}


TABLE 7. Gait Characteristics

\begin{tabular}{|c|c|c|c|c|c|c|}
\hline Characteristic & Pre-test & Day 1 & Day 2 & Day 3 & Post-test & $\begin{array}{l}\text { Magnitude } \\
\text { of change }\end{array}$ \\
\hline \multicolumn{7}{|l|}{ Stride length (cm) } \\
\hline Right & 35.98 & 33.52 & 38.54 & 44.16 & 40.58 & 5.27 \\
\hline Left & 35.58 & 33.88 & 38.98 & 43.67 & 40.58 & 4.6 \\
\hline Cadence (steps/min) & 49.2 & 55.3 & 56.2 & 54.1 & 53.6 & 4.4 \\
\hline Velocity (m/s) & 14.7 & 15.6 & 18 & 19.7 & 18.2 & 3.5 \\
\hline Ambulation time (s) & 25.63 & 23.87 & 21.37 & 19.95 & 21.27 & $(-) 4.36$ \\
\hline
\end{tabular}

TABle 8. Percentage of Swing and Stance Phase of Gait

\begin{tabular}{|c|c|c|c|c|c|c|}
\hline Leg & Pre-test & Day 1 & Day 2 & Day 3 & Post-test & $\begin{array}{c}\text { Change } \\
\text { (post-pre) }\end{array}$ \\
\hline \multicolumn{7}{|l|}{ Right } \\
\hline Swing (\%) & 15.7 & 17.9 & 19.9 & 21.0 & 20.3 & 4.6 \\
\hline Stance (\%) & 84.3 & 82.1 & 80.1 & 79.0 & 79.7 & $(-) 4.6$ \\
\hline \multicolumn{7}{|l|}{ Left } \\
\hline Swing (\%) & 11.6 & 12 & 14.4 & 14.4 & 14.2 & 2.6 \\
\hline Stance $(\%)$ & 88.4 & 88 & 85.6 & 85.6 & 85.8 & $(-) 2.6$ \\
\hline
\end{tabular}

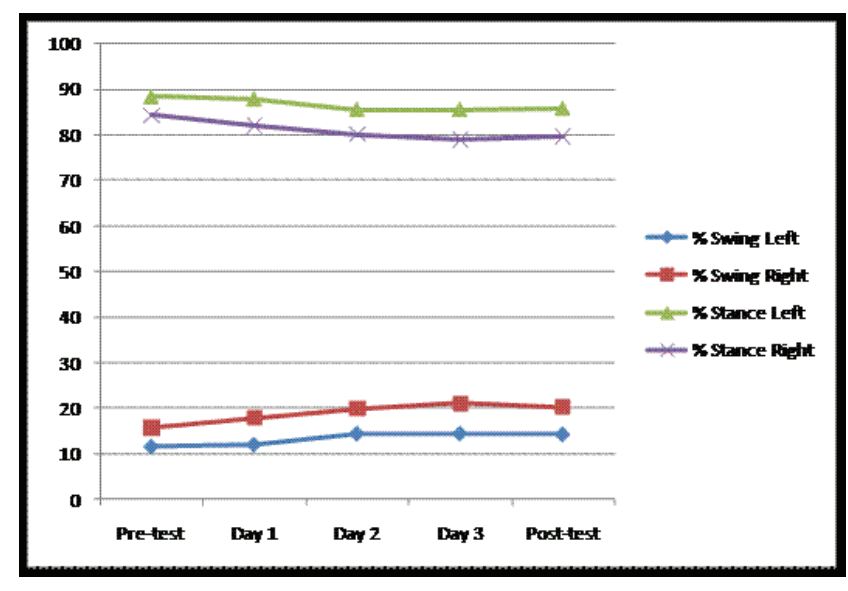

FIGURE 2. Swing and stance phase changes.

\section{DISCUSSION}

The results of the present study suggest that specific routines of massage therapy may have had an effect on ROM and gait characteristics within an allotted timeframe post treatment for this particular participant with incomplete SCI.

The authors hypothesized that reduction of trigger points, combined with the passive stretch effects of massage strokes, would help to restore the proper length-tension relationship in the treated muscle groups, leading to ROM improvements. Results for ROM varied across test sessions in this participant, with positive signs for hip extension measures, negative signs for SLR, and mixed results for DF from pre to post in all interventions.

The authors expected that increases in ROM would correlate with treatment trial. Specifically, they expected that the hamstring routine would lead to increased SLR measurement, that the triceps surae treatment would lead to increases in dorsiflexor length, and that iliopsoas treatment would increase hip extension ROM, with the greatest increase in ROM therefore demonstrated on the respective intervention day. Overall post-treatment gains in bilateral hip extension and left DF were observed; however, correlations between a specific routine and a specific ROM gain were not observed.

The authors further hypothesized that spasticity would be temporarily reduced within a short timeframe post intervention. However, no significant changes in spasticity were noted as measured by the MAS. Several factors could have affected this outcome. First, spasticity has three known components, and the MAS measures only one. Specifically, the MAS measures only resistance to passive movement. The spasticity syndrome, often seen in incomplete SCI (and in other neurologic injuries), also often includes an exaggerated component of the stretch reflex. Hyperexcitability of this tonic reflex can result in increased muscle tone in response to passive stretch because of disinhibition of descending tracks from upper motor neuron lesions ${ }^{(41)}$.

Extrinsic spasticity-defined as spasm caused by perceived noxious stimuli $(34,42) \ldots$ is not captured by the MAS. It is therefore possible that change in 
some components of spasticity was not measured in the present study and that a more specific tool is needed to evaluate changes potentially caused by the intervention. Although some authors find value in the MAS $^{(43)}$, Adams and colleagues ${ }^{(44)}$ suggest that use of the Spinal Cord Assessment Tool for Spasticity Reflexes or the National Institute of Neurological Disorders and Stroke Myotatic Reflex Scale (or both) may provide a more comprehensive evaluation of spasticity, especially when combined with additional participant and objective assessments.

Another consideration of why changes in spasticity were not noted is that the MAS may not be a valid tool. A criticism of the MAS is its inability to distinguish between tonal properties and soft-tissue restrictions $^{(3,45)}$. The inter-rater reliability of MAS is also a concern ${ }^{(46)}$. Two PTs trained in application of MAS conducted the tests for the present study; however, it is possible that the inter-rater reliability was poor.

The study participant demonstrated changes in gait characteristics during the treatment period. Specifically, he demonstrated an increase in stride length, velocity, and cadence; improvements in swing-tostance time ratios for gait cycle; and a decrease in ambulation time. Pepin and colleagues ${ }^{(47,48)}$ suggest that increases in stride length and frequency (cadence) are the prime drivers for adaptation to walking speed in the able-bodied population. In the study participant, speed-related parameters (ambulation time, velocity, cadence), stride length, and swing and stance percentages were all interrelated. As velocity increased, ambulation time declined (as it would in any person). Cadence (stride frequency) is affected not only by stride length, but also by swing and stance times. Normal ratio estimates for swing and stance times are approximately 32\% swing and 68\% stance $^{(9,49)}$, and the study participant demonstrated a positive trend in those ratios (Table 8). Pepin et al. ${ }^{(48)}$ showed increased double-limb support time and an inability to increase stride length and frequency as limiting factors in achieving adaptation to speed in people post SCI. Van Hedel and colleagues ${ }^{(7,8)}$ further concluded that adaptations to walking speeds are achieved by an increase in cadence and stride length, often simultaneously. Further, an inability to increase those parameters was shown to be the limiting factor to increased walking speeds in the SCI population.

These gait characteristic changes, when coupled with the study participant's report of ease of movement 5 days post intervention, suggest a possible improvement in firing patterns or muscle activation (or both) post massage. One explanation for the changes is that massage may have led to reduced motor neuron activity and excitability, and therefore allowed for greater muscle activation. However, another explanation is that an interaction between musculoskeletal effects and neural impairments resulted in the changes in gait characteristics.
Increases in stride length may have resulted from a number of factors, although some of those factors remain speculative. Enhanced iliopsoas firing may have resulted in increased stride length by improving hip flexion motion. Reduction of iliopsoas tightness could allow greater hip extension ROM and improve activation of gluteus maximus, its antagonist, through reciprocal inhibition. More efficient firing of the gluteus maximus can allow for greater stance time and longer swing on the contralateral side.

Through temporary disinhibition of hamstrings or relaxation and lengthening of the hamstring group (or both), quadriceps femoris activation can also allow for an increase in stride length. Notably, the largest gains in stride length were observed after the hamstring treatment. Hopper et al. ${ }^{(50)}$ showed an increase in hamstring length with dynamic soft-tissue mobilization in healthy male participants; however, this passive length increase was not compared with gait stride length nor with a neurologically impaired population.

Improved activation of pre-tibial musculature through disinhibition of the triceps surae group can allow for improved foot clearance in swing and tibial motion. Improved tibial motion control may allow greater swing time on the contralateral limb. Other studies have demonstrated the importance of maintaining the gastrocnemius-soleus group length, given its changes in length-tension relationship ${ }^{(51)}$ after SCI and its consequential effect on gait.

Increases in cadence and velocity, decreases in ambulation time, and improvements in swing-to-stance ratios in the gait cycle were notable and should be explored further, given that gait function improvements in those areas can have a great influence on level of independence in a person with SCI.

Improvements in gait parameters aside from cadence showed a commonality, in that day 3 showed the most change universally. On that day, the hamstring routine might have had unique qualities, or conversely, a cumulative effect may have resulted from the iliopsoas and triceps surae group massages that were applied on the preceding days. Notably, no measure returned to baseline on the post-intervention day, when no treatment was applied, suggesting a carryover effect for 24 hours. We would suggest retesting the participant at intervals after 24 hours to see if and when a return to baseline occurs.

Other explanations for the changes in the gait characteristics may include other non-neural and non-muscular mechanisms. Our study did not address the possible effects of massage on vasodilation, connective tissue plasticity, or force transmission. People with SCI have impaired vascular control ${ }^{(52,53)}$. Massage may temporarily influence blood flow by stimulating vasodilation through activation of parasympathetic pathways to local musculature ${ }^{(54-56)}$. However, the effect of blood flow in response to massage in incomplete SCI has not been studied, and this hypothesis would need to be further explored. 
Although our massage techniques were not specifically directed to the connective tissue, Kassolik and colleagues ${ }^{(57,58)}$ found that a muscle connected indirectly by structural elements to a muscle being massaged can demonstrate electrical and mechanical activity. The massage intervention provided in the present study may have influenced connective tissue elements according to the theory of tensegrity $(58,59)$. Connective tissue structures undergo changes - such as accumulation of collagen and adipocytes ${ }^{(4)}$ - in relation to SCI. Subclinical cellular changes in intrinsic passive stiffness not related to neural mechanisms have also been explored ${ }^{(3,60,61)}$. Although we hypothesize that these structures were also receiving sensory input during massage, the effect is unknown and unmeasured. The effect of massage on these altered structures is also speculative.

The role of connective tissue in transmission of force, including the effect on synergist and antagonist movement and lateral force transmission, are the focus of several investigations ${ }^{(62,63)}$. Huijing $^{(64)}$ and Van der $\mathrm{Wal}^{(65)}$ report a role for connective tissue in motor control and proprioception. Schleip (alone and with various colleagues) ${ }^{(66-70)}$ has also presented the theory that fascia is able to contract in a smooth-musclelike manner, therefore possibly influencing passive muscle stiffness. These new theories of connective tissue function have implications for the possible influence of massage on muscle function, but they are as yet untested. We therefore do not know what, if any, influence massage may have on these forces and, therefore, function.

\section{Study Limitations}

Our study has some limitations. For instance, measures of passive ROM were taken in the standard position and using the protocol found in Clarkson ${ }^{(71)}$. However, test position stabilization may have affected results in this area. By having healthy participants don a knee extension brace, ankle-foot orthosis, and hip-stabilizing belt, Hopper et al. ${ }^{(50)}$ allowed for more specific control over knee and ankle motion in their studies measuring SLR. In those studies, hamstring flexibility was defined as the difference between total range of SLR and range of pelvic rotation. The lack of external hip stabilization, combined with manual-only control of knee and hip position, may have affected our outcomes for SLR measurement.

Furthermore, passive ROM measurements showed variability when the examiner changed on day 3 . That change, and not the intervention, may account for the changes in ROM observed on that day. In a cerebral palsy population, Mutlu and co-workers ${ }^{(72)}$ demonstrated inter- and intra-testing reliability in ROM measurements for multiple PTs, but a PubMed search uncovered no comparable study for SCI participants. The reliability of goniometric ROM measurements has been established for other patient populations, but more research is needed to examine measurement reliability in the SCI population. In addition, passive ROM testing in the supine position may not correlate with active ROM during gait. Our study participant may have achieved greater active ROM during gait that was not reflected in the passive ROM measures.

Our study participant did not show changes in MAS, a result that may have been related to test specificity or multiple examiners. Future studies should incorporate the use of a more sensitive spasticity measure and a self-report mechanism for functional changes post massage.

In this application of massage, a set routine was applied by the same MT throughout the study; however, the stroke pressure and speed were not controlled. Future studies should attempt to control for these factors, given that they can affect outcome ${ }^{(26,73)}$.

Finally, to monitor the consistency of any changes post massage, future studies should also include outcome assessments at time points beyond 24 hours post intervention.

\section{CONCLUSIONS}

Positive changes in gait speed, stride length, and swing and stance phase percentages were observed in one person with incomplete AIS(D) SCI after specific application of MT. Further study is warranted to determine the extent to which massage may affect musculoskeletal and neural impairments that limit gait in people with incomplete SCI, and the methods or routines that, when applied, will yield the most benefit.

\section{CONFLICT OF INTEREST NOTIFICATION}

The authors declare that there are no conflicts of interest.

\section{COPYRIGHT}

Published under the CreativeCommons AttributionNonCommercial-NoDerivs 3.0 License.

\section{REFERENCES}

1. National Spinal Cord Injury Statistical Center (NSCISC). Spinal Cord Injury Facts and Figures at a Glance. NSCISC website. https://www.nscisc.uab.edu/public content/pdf/FactsApr09. pdf. Published April 2009. Accessed June 1, 2009.

2. Teixeira da Cunha-Filho I, Henson H, Qureshy H, Williams AL, Holmes SA, Protas EJ. Differential responses to measures of gait performance among healthy and neurologically impaired individuals. Arch Phys Med Rehabil. 2003; 84(12):1774-1779. 
3. Gracies JM. Pathophysiology of spastic paresis. I: Paresis and soft tissue changes. Muscle Nerve. 2005; 31(5):535-551.

4. Biering-Sørensen B, Kristensen IB, Kjaer M, Biering-Sørensen F. Muscle after spinal cord injury. Muscle Nerve. 2009; 40(4):499-519.

5. van der Salm A, Nene AV, Maxwell DJ, Veltink PH, Hermens HJ, IJzerman MJ. Gait impairments in a group of patients with incomplete spinal cord injury and their relevance regarding therapeutic approaches using functional electrical stimulation. Artif Organs. 2005; 29(1):8-14.

6. Kim CM, Eng JJ, Whittaker MW. Level walking and ambulatory capacity in persons with incomplete spinal cord injury: relationship with muscle strength. Spinal Cord. 2004; 42(3):156-162.

7. van Hedel HJ, Dietz V, Curt A. Assessment of walking speed and distance in subjects with an incomplete spinal cord injury. Neurorehabil Neural Repair. 2007; 21(4):295-301.

8. van Hedel HJ on behalf of the EMSCI Study Group. Gait speed in relation to categories of functional ambulation after spinal cord injury. Neurorehabil Neural Repair. 2009; 23(4):343-50.

9. Rancho Los Amigos National Rehabilitation Center, Pathokinesiology Service and Physical Therapy Department. Observational Gait Analysis. 4th ed. Downey, CA: Los Amigos Research and Education Institute, Rancho Los Amigos National Rehabilitation Center; 2001.

10. Perry J, Burnfield JM. Gait Analysis: Normal and Pathological Function. 2nd ed. Thorofare, NJ: Slack; 2010.

11. Chaitow L, DeLany J. Clinical Application of Neuromuscular Techniques. Vol. 2. The Lower Body. Edinburgh, UK: Churchill Livingstone; 2002.

12. Jurch SE. Clinical Massage Therapy: Assessment and Treatment of Orthopedic Conditions. Boston, MA: McGraw-Hill Higher Education; 2009.

13. Hedrickson T. Massage and Manual Therapy for Orthopedic Conditions. 2nd ed. Philadelphia, PA: Lippincott, Williams and Wilkins; 2009.

14. Lowe WW. Orthopedic Assessment in Massage Therapy. Sisters, OR: Daviau-Scott; 2006.

15. Brooks CP, Woodruff LD, Wright LL, Donatelli R. The immediate effects of manual massage on power-grip performance after maximal exercise in healthy adults. J Altern Complement Med. 2005; 11(6):1093-1101.

16. Ogai R, Yamane M, Matsumoto T, Kosaka M. Effects of petrissage massage on fatigue and exercise performance following intensive cycle pedalling. Br J Sports Med. 2008; 42(10):834-838.

17. Dryden T, Baskwill A, Preyde M. Massage therapy for the orthopaedic patient: a review. Orthop Nurs. 2004; 23(5):327-334.

18. Best TM, Hunter R, Wilcox A, Haq F. Effectiveness of sports massage for recovery of skeletal muscle from strenuous exercise. Clin J Sports Med. 2008; 18(5):446-460.

19. Furlan AD, Imamura M, Dryden T, Irvin E. Massage for low back pain: an updated systematic review within the framework of the Cochrane Back Review Group. Spine (Phila Pa 1976). 2009; 34(16):1669-1684.

20. Nayak S, Matheis RJ, Agostinelli S, Shifleft SC. The use of complementary and alternative therapies for chronic pain following spinal cord injury: a pilot survey. J Spinal Cord Med. 2001; 24(1):54-62.
21. Norrbrink Budh C, Lundeberg T. Non-pharmacological pain-relieving therapies in individuals with spinal cord injury: a patient perspective. Complement Ther Med. 2004; 12(4):189-197.

22. Widerström-Noga EG, Turk DC. Types and effectiveness of treatments used by people with chronic pain associated with spinal cord injuries: influence of pain and psychosocial characteristics. Spinal Cord. 2003; 41(11):600-609.

23. Diego MA, Field T, Hernandez-Reif M, Hart S, Brucker B, Field $\mathrm{T}$, et al. Spinal cord patients benefit from massage therapy. Int J Neurosci. 2002; 112(2):133-142.

24. Morelli M, Seaborne DE, Sullivan SJ. H-Reflex modulation during manual muscle massage of human triceps surae. Arch Phys Med Rehabil. 1991; 72(11):915-919.

25. Sullivan SJ, Williams LR, Seaborne DE, Morelli M. Effects of massage on alpha motoneuron excitability. Phys Ther. 1991; 71(8):555-560.

26. Morelli M, Chapman CE, Sullivan SJ. Do cutaneous receptors contribute to the changes in the amplitude of the H-reflex during massage? Electromyogr Clin Neurophysiol. 1999; 39(7):441-447.

27. Goldberg J, Seaborne DE, Sullivan SJ, Leduc BE. The effect of therapeutic massage on H-reflex amplitude in persons with a spinal cord injury. Phys Ther. 1994; 74(8):728-737.

28. Brouwer B, Sousa de Andrade V. The effects of slow stroking on spasticity in patients with multiple sclerosis: a pilot study. Physiother Theory Pract. 1995; 11(1):13-21.

29. Macgregor R, Campbell R, Gladden MH, Tennant N, Young D. Effects of massage on the mechanical behaviour of muscles in adolescents with spastic diplegia: a pilot study. Dev Med Child Neurol. 2007; 49(3):187-191.

30. Hernandez-Reif M, Field T, Largie S, Diego M, Manigat N, Seoanes J, et al. Cerebral palsy symptoms in children decreased following massage therapy. Early Child Dev Care. 2005; 175(5):445-456.

31. Lucas K, Polus B, Rich P. Latent myofascial trigger points: their effects on muscle activation and movement efficiency. $J$ Bodyw Mov Ther. 2004; 8:160-166.

32. Lucas KR. The impact of latent trigger points on regional muscle function. Curr Pain Headache Rep. 2008; 12(5):344-349.

33. Hong CZ, Simons DG. Pathophysiologic and electrophysiologic mechanisms of myofascial trigger points. Arch Phys Med Rehabil. 1998; 79(7):863-872.

34. Mirbagheri MM, Barbeau H, Ladouceur M, Kearney RE. Intrinsic and reflex stiffness in normal and spastic, spinal cord injured subjects. Exp Brain Res. 2001; 141(4):446-459.

35. American Spinal Injury Association. Reference Manual of the International Standards for Neurological Classification of Spinal Cord Injury. Chicago, IL: American Spinal Injury Association; 2003.

36. Sisto SA, Druin E, Macht Sliwinski M. Spinal Cord Injuries: Management and Rehabilitation. St. Louis, MO: Mosby Elsevier; 2009: p. 27.

37. Craven BC, Morris AR. Modified Ashworth scale reliability for measurement of lower extremity spasticity among patients with SCI. Spinal Cord. 2010; 48(3):207-213.

38. Karin Harms-Ringdahl, ed. Muscle Strength. New York, NY: Churchill Livingstone; 1993.

39. Kostopoulos D, Rizopoulos K. The Manual of Trigger Point and Myofascial Therapy. Thorofare, NJ: Slack; 2001: p. 3-31. 
40. Turchaninov R. Therapeutic Massage: A Scientific Approach. Phoenix, AZ: Aesculapius; 2000.

41. Adams MM, Hicks AL. Spasticity after spinal cord injury. Spinal Cord. 2005; 43(10):577-586.

42. Sheean G. The pathophysiology of spasticity. Eur J Neurol. 2002; 9(Suppl 1):3-9,53-61.

43. Tederko P, Krasuski M, Czech J, Dargiel A, Garwacka-Jodzis I, Wojciechowska A. Reliability of clinical spasticity measurements in patients with cervical spinal cord injury. Ortop Traumatol Rehabil. 2007; 9(5):467-483.

44. Adams MM, Ginis KA, Hicks AL. The Spinal Cord Injury Spasticity Evaluation Tool: development and evaluation. Arch Phys Med Rehabil. 2007; 88(9):1185-1192.

45. Priebe MM. Assessment of spinal cord injury spasticity in clinical trials. Top Spinal Cord Inj Rehabil. 2006; 11(3):69-77.

46. Haas BM, Bergström E, Jamous A, Bennie A. The inter rater reliability of the original and of the modified Ashworth scale for the assessment of spasticity in patients with spinal cord injury. Spinal Cord. 1996; 34(9):560-564.

47. Pépin A, Norman KE, Barbeau H. Treadmill walking in incomplete spinal-cord-injured subjects: 1 . Adaptation to changes in speed. Spinal Cord. 2003; 41(5):257-270.

48. Pépin A, Ladouceur M, Barbeau H. Treadmill walking in incomplete spinal-cord-injured subjects: 2 . Factors limiting the maximal speed. Spinal Cord. 2003; 41(5):271-279.

49. Chambers HG, Sutherland DH. A practical guide to gait analysis. J Am Acad Orthop Surg. 2002; 10(3):222-231.

50. Hopper D, Deacon S, Das S, Jain A, Riddell D, Hall T, et al. Dynamic soft tissue mobilisation increases hamstring flexibility in healthy male subjects. Br J Sports Med. 2005; 39(9):594-398.

51. Pelletier CA, Hicks AL. The length-tension relationship of human dorsiflexor and plantarflexor muscles after spinal cord injury. Spinal Cord. 2010; 48(3):202-206.

52. Olive JL, McCully KK, Dudley GA. Blood flow response in individuals with incomplete spinal cord injuries. Spinal Cord. 2002; 40(12):639-645.

53. Olive JL, Dudley GA, McCully KK. Vascular remodeling after spinal cord injury. Med Sci Sports Exerc. 2003; 35(6):901-907.

54. Mori H, Ohsawa H, Tanaka TH, Taniwaki E, Leisman G, Nishijo K. Effect of massage on blood flow and muscle fatigue following isometric lumbar exercise. Med Sci Monit. 2004; 10(5):CR173-CR178.

55. Hinds T, McEwan I, Perkes J, Dawson E, Ball D, George K. Effects of massage on limb and skin blood flow after quadriceps exercise. Med Sci Sports Exerc. 2004; 36(8):1308-1313.

56. Takamoto K, Sakai S, Hori E, Urakawa S, Umeno K, Ono T, et al. Compression on trigger points in the leg muscle increases parasympathetic nervous activity based on heart rate variability. J Physiol Sci. 2009; 59(3):191-197.

57. Kassolik K, Andrzejewski W, Trzęsicka E. Role of the tensegrity rule in theoretical basis of massage therapy. J Back Musculoskelet Rehabil. 2007; 20(1):15-20.

58. Kassolik K, Jaskólska A, Kisiel-Sajewicz K, Marusiak J, Kawczyński A, Jaskólski A. Tensegrity principle in massage demonstrated by electro- and mechanomyography. J Bodyw Mov Ther. 2009; 13(2):164-170.

59. Chaudhry H, Schleip R, Ji Z, Bukiet B, Maney M, Findley T. Three-dimensional mathematical model for deformation of human fasciae in manual therapy. J Am Osteopath Assoc. 2008; 108(8):379-390.

60. Olsson MC, Krüger M, Meyer LH, Ahnlund L, Gransberg L, Linke WA, et al. Fibre type-specific increase in passive muscle tension in spinal cord-injured subjects with spasticity. JPhysiol. 2006; 577(Pt 1):339-352.

61. Dietz V, Sinkjaer T. Spastic movement disorder: impaired reflex function and altered muscle mechanics. Lancet Neurol. 2007; 6(8):725-733.

62. Huijing PA. Epimuscular myofascial force transmission between antagonistic and synergistic muscles can explain movement limitation in spastic paresis. J Electromyogr Kinesiol. 2007; 17(6):708-724.

63. Shinohara M, Yoshitake Y, Kouzaki M. Alterations in synergistic muscle activation impact fluctuations in net force. Med Sci Sports Exerc. 2009; 41(1):191-197.

64. Huijing PA. Muscular force transmission necessitates a multilevel integrative approach to the analysis of function of skeletal muscle. Exerc Sport Sci Rev. 2003; 31(4):167-175.

65. van der Wal J. The architecture of the connective tissue in the musculoskeletal system - an often overlooked functional parameter as to proprioception in the locomotor apparatus. Int J Ther Massage Bodyw. 2009; 2(4):9-23. http://journals.sfu.ca/ ijtmb/index.php/ijtmb/article/view/62/79. Published December 2009. Accessed June 1, 2009.

66. Schleip R. Fascial plasticity - a new neurobiological explanation: part 1. J Bodyw Mov Ther. 2003; 7(1):11-19.

67. Schleip R. Fascial plasticity - a new neurobiological explanation: part 2. J Bodyw Mov Ther. 2003; 7(2):104-116.

68. Schleip R, Klingler W, Lehmann-Horn F. Active contraction of the thoracolumbar fascia-indications of a new factor in low back pain research with implications for manual therapy. In: Vleeming A, Mooney V, Hodges P, eds. Proceedings of the Fifth Interdisciplinary World Congress on Low Back and Pelvic Pain. Melbourne, Australia: Fascia Research Project; 2004.

69. Schleip R, Klingler W, Lehmann-Horn F. Active fascial contractility: fascia may be able to contract in a smooth muscle-like manner and thereby influence musculoskeletal dynamics. Med Hypotheses. 2005; 65(2):273-277.

70. Schleip R, Naylor IL, Ursu D, Melzer W, Zorn A, Wilke HJ, et al. Passive muscle stiffness may be influenced by active contractility of intramuscular connective tissue. Med Hypotheses. 2006; 66(1):66-71.

71. Clarkson HM. Joint Motion and Function Assessment: A Research-based Practical Guide. Philadelphia, PA: Lippincott, Williams and Wilkins; 2005.

72. Mutlu A, Livanelioglu A, Gunel MK. Reliability of goniometric measurements in children with spastic cerebral palsy. Med Sci Monit. 2007; 13(7):CR323-CR329.

73. Goldberg J, Sullivan SJ, Seaborne DE. The effect of two intensities of massage on H-reflex amplitude. Phys Ther. 1992; 72(6):449-457.

Corresponding author: Christine Manella, Spinal Cord Injury Program, Shepherd Center, 2020 Peachtree Road, Atlanta, Georgia 30309 USA.

E-mail: christine_manella@shepherd.org 